\title{
Thyroid Parameters and Kidney Disorder in Type 2 Diabetes: Results from the METAL Study
}

\author{
Yi Chen $(\mathbb{D}$, Wen Zhang, Ningjian Wang $(\mathbb{D}$, Yuying Wang, Chiyu Wang, Heng Wan, \\ and Yingli Lu \\ Institute and Department of Endocrinology and Metabolism, Shanghai Ninth People's Hospital, Shanghai Jiao Tong University School \\ of Medicine, Shanghai, China \\ Correspondence should be addressed to Yingli Lu; luyingli2008@126.com
}

Received 7 January 2020; Revised 3 March 2020; Accepted 9 March 2020; Published 30 March 2020

Academic Editor: Ferdinando Carlo Sasso

Copyright ( 2020 Yi Chen et al. This is an open access article distributed under the Creative Commons Attribution License, which permits unrestricted use, distribution, and reproduction in any medium, provided the original work is properly cited.

\begin{abstract}
Objective. Diabetic kidney disease is one of the most common microvascular complications of diabetes mellitus. We aimed to analyze the association of thyroid parameters with kidney disorders, especially in euthyroid participants. Methods. The data were obtained from a cross-sectional study, the METAL study. Thyroid parameters, including thyroid-stimulating hormone (TSH), free triiodothyronine $\left(\mathrm{FT}_{3}\right)$, free thyroxine $\left(\mathrm{FT}_{4}\right)$, triiodothyronine $\left(\mathrm{T}_{3}\right)$, thyroxin $\left(\mathrm{T}_{4}\right)$, thyroid peroxidase antibody (TPOAb), and thyroglobulin antibody (TgAb), of 4136 participants with type 2 diabetes were measured. Two structure parameters of thyroid homeostasis, including the sum activity of step-up deiodinases (SPINA-G $\mathrm{G}_{\mathrm{D}}$ ) and thyroid secretory capacity (SPINA- $\mathrm{G}_{\mathrm{T}}$ ), and two pituitary thyrotropic function indices, including Jostel's TSH index (TSHI) and the thyrotroph thyroid hormone resistance index (TTSI), were also calculated. Kidney disorders were described according to the presence of reduced estimated glomerular filtration rate (eGFR) and/or higher urinary albumin to creatinine ratio (UACR). Results. The prevalence of kidney disorders increased with decreasing $\mathrm{FT}_{3}$ or $\mathrm{T}_{3}$ and increasing $\mathrm{FT}_{4}$ or $\mathrm{T}_{4}$ quartile levels (all $P<0.05$ ). After full adjustment, linear regression showed that UACR levels were negatively associated with $\mathrm{FT}_{3}$ and $\mathrm{T}_{3}(P<0.001)$. In addition, eGFR was positively associated with $\mathrm{FT}_{3}$ and $\mathrm{T}_{3}$ and was negatively associated with $\mathrm{TSH}$ and $\mathrm{FT}_{4}$ levels and TgAb positivity (all $P<0.05$ ). By using binary logistic regression, higher TSH and $\mathrm{FT}_{4}$ and lower $\mathrm{FT}_{3}$ and $\mathrm{T}_{3}$ were associated with kidney disorders (all $P<0.05$ ). Similar results were seen in sensitivity analyses, which were performed in 3035 euthyroid diabetic participants; however, TSH was no longer related to them. The area under the receiver operating characteristic curve (AUROC) of lower FT 3 for existing kidney disorder was greater than that for any other thyroid hormones (all $P<0.001$ ). The cutoff value of $\mathrm{FT}_{3}$ for reduced eGFR was $4.39 \mathrm{pmol} / \mathrm{L}$. Regarding thyroid homeostasis parameters, SPINA-G $\mathrm{D}_{\mathrm{D}}$ was negatively associated with three statuses of kidney disorders, and TSHI and TTSI were positively associated with reduced eGFR (all $P<0.05$ ). Conclusions. Among patients with type 2 diabetes, elevated TSH and $\mathrm{FT}_{4}\left(\right.$ or $\mathrm{T}_{4}$ ), lower $\mathrm{FT}_{3}\left(\right.$ or $\mathrm{T}_{3}$ ), TgAb positivity, lower SPINA-G $\mathrm{G}_{\mathrm{D}}$, and higher TSHI and TTSI were associated with kidney disorders. The lower $\mathrm{FT}_{3}$, even within the normal range $(<4.38 \mathrm{pmol} / \mathrm{L})$, may be the factor most related to reduced eGFR compared with other thyroid hormones in diabetic patients.
\end{abstract}

\section{Introduction}

Diabetes mellitus (DM) and its complications have become highly prevalent and have gained increasing attention [1], especially in developing countries. The estimated prevalence of diabetes among a representative sample of Chinese adults was $11.6 \%$, which indicates the importance of diabetes as a public health problem in China [2]. Chronic kidney disease (CKD) is defined by the sustained presence of reduced kidney function or damage, often resulting from diabetes mellitus (DM) and hypertension [3]. Approximately 13\% of individuals in the general US population have CKD, and the inci- 
dence is increasing globally [4-6]. Diabetic kidney disease (DKD) is one of the most common microvascular complications of diabetes mellitus and the leading cause of end-stage renal disease (ESRD) worldwide [7]. Approximately 20\%$40 \%$ of patients with diabetes progress to DKD, and $40 \%$ also progress to ESRD [8]. Reduced estimated glomerular filtration rate (eGFR) and higher urinary albumin to creatinine ratio (UACR) levels are two main markers of diabetic kidney status, quantifying renal function and serving as a proxy of renal damage severity, respectively $[3,9]$.

The thyroid gland is one of the most important organs in the human body. It regulates the majority of the body's physiological actions [10]. Thyroid hormone has an impact on renal tubular function and the renin-angiotensin system and is associated with hemodynamic and cardiovascular alterations that interfere with renal blood flow [11]. Conversely, the kidney is not only an organ for the metabolism and elimination of $\mathrm{TH}$ but also a target organ of some of the actions of the iodothyronines [12]. Acute kidney injury and chronic kidney disease are accompanied by notable effects on the hypothalamus-pituitary-thyroid axis. Hormonal derangements at the level of the hypothalamicpituitary axis are often seen with the worsening of kidney function, and recent evidence points towards the implication of such hormonal disorders in the genesis of CKD [13]. Thyroid dysfunction causes remarkable changes in glomerular and tubular functions and in electrolyte and water homeostasis [12].

Although several studies have suggested the association of thyroid disorders and CKD or DKD with conflicting results $[7,14-16]$, studies focusing on the possible relationship between thyroid status and eGFR and UACR among a relative number of DM participants, especially in euthyroid DM patients, have been limited. The present cross-sectional study investigated whether thyroid parameter concentrations, including thyroid-stimulating hormone (TSH), free triiodothyronine $\left(\mathrm{FT}_{3}\right)$, free thyroxine $\left(\mathrm{FT}_{4}\right), \mathrm{T}_{3}, \mathrm{~T}_{4}$, thyroid peroxidase antibody (TPOAb), and thyroglobulin antibody $(\mathrm{TgAb})$, and four parameters of thyroid homeostasis, including the sum activity of step-up deiodinases (SPINA- $\mathrm{G}_{\mathrm{D}}$ ), thyroid secretory capacity (SPINA- $\mathrm{G}_{\mathrm{T}}$ ), Jostel's TSH index (TSHI), and thyrotroph thyroid hormone resistance index (TTSI), were related to eGFR, UACR, and the prevalence of kidney disorders, described as reduced eGFR, higher UACR, and higher UACR and/or reduced eGFR in type 2 diabetes.

\section{Methods}

2.1. Study Design and Participants. The data of this study were obtained from a cross-sectional study, the METAL study (Environmental Pollutant Exposure and Metabolic Diseases in Shanghai, http://www.chictr.org.cn, ChiCTR1800017573) $[17,18]$, which was designed to investigate the association between exposure to heavy metals and diabetic complications in Chinese diabetic adults. We enrolled participants from seven communities in the Huangpu and Pudong districts in Shanghai. In 2018, we obtained the list of diabetic patients who were Chinese citizens $\geq 18$ years old and had lived in their current area for $\geq 6$ months from the registration plat- form in each community healthcare center and then randomly selected $50 \%$ of them $(n=4937)$ to receive the examination by using SPSS Statistics, Version 22 (IBM Corporation, Armonk, NY, USA). We excluded participants who were missing laboratory results $(n=8)$ or questionnaire data $(n=116)$, those who were missing thyroid parameters $(n=6)$ or UACR results $(n=234)$, those having a history of thyroid surgery or thyroid disorder treatment $(n=265)$, or those having a history of glucocorticoid or amiodarone treatment $(n=172)$ in this study. Finally, 4136 participants were involved in this study. Then, we excluded subjects with abnormal $\mathrm{TSH}, \mathrm{FT}_{4}$, or $\mathrm{T}_{4}$ levels and those with $\mathrm{FT}_{3}$ or $\mathrm{T}_{3}$ levels below the lower limits of their reference ranges for sensitivity analysis (Figure 1). All participants provided written informed consent before data collection. The study protocol was approved by the Ethics Committee of Shanghai Ninth People's Hospital, Shanghai Jiao Tong University School of Medicine. All procedures followed were in accordance with the ethical standards of the responsible committee on human experimentation (institutional and national) and with the 1975 Declaration of Helsinki, as revised in 2008.

2.2. Laboratory Measurements. Serum samples for laboratorial assays were obtained by venipuncture after an 8 -hour fast. Blood samples were stored at $-20^{\circ} \mathrm{C}$ when collected and shipped by air in dry ice to one central laboratory, which was certified by the College of American Pathologists (CAP), within 2-4 hours of collection.

Serum TSH, $\mathrm{FT}_{3}, \mathrm{FT}_{4}, \mathrm{~T}_{3}, \mathrm{~T}_{4}, \mathrm{TPOAb}$, and $\mathrm{TgAb}$ were measured by electrochemiluminescence (Roche, E601, Germany). The normal reference ranges for $\mathrm{TSH}, \mathrm{FT}_{3}, \mathrm{FT}_{4}$, $\mathrm{T}_{3}, \mathrm{~T}_{4}$, TPOAb, and $\mathrm{TgAb}$ were $0.27-4.20 \mathrm{mIU} / \mathrm{L}, 3.10$ $6.80 \mathrm{pmol} / \mathrm{L}, 12.00-22.00 \mathrm{pmol} / \mathrm{L}, 1.30-3.10 \mathrm{nmol} / \mathrm{L}, 66.00-$ $181.00 \mathrm{nmol} / \mathrm{L}, 0-34.00 \mathrm{U} / \mathrm{mL}$, and $0-115.00 \mathrm{U} / \mathrm{mL}$, respectively. Euthyroid was defined as $\mathrm{TSH}, \mathrm{FT}_{4}$, and $\mathrm{T}_{4}$ levels within normal limits and without $\mathrm{FT}_{3}$ or $\mathrm{T}_{3}$ levels below the lower limits. Two structure parameters of thyroid homeostasis, SPINA- $\mathrm{G}_{\mathrm{D}}$ and SPINA- $\mathrm{G}_{\mathrm{T}}$, as well as two pituitary thyrotropic function indices, TSHI and TTSI, were calculated according to previous studies [19-21].

Creatinine (Cr) and uric acid (UA) were measured by Beckman Coulter AU680 (Brea, USA). Using morning fasting spot urine samples, the concentrations of albumin and CR were measured with a Beckman Coulter AU680 (Brea, USA) using a turbidimetric immunoassay and an enzymatic method, respectively. Reduced eGFR was defined as eGFR $<60 \mathrm{~mL} / \mathrm{min} / 1.73 \mathrm{~m}^{2}$ according to the Chronic Kidney Disease Epidemiology Collaboration (CKD-EPI) for "Asian origin" [22] and regardless of whether the UACR was high. Higher UACR was defined as UACR $\geq 30 \mathrm{mg} / \mathrm{g}$, suggested by an ADA statement [23] and regardless of the value of eGFR. Higher UACR and/or reduced eGFR was defined as the positivity of higher UACR and/or reduced eGFR.

Lipid profiles, including triglycerides (TG), total cholesterol (TC), and high- (HDL-C) and low-density lipoprotein (LDL-C), were measured by Beckman Coulter AU680 (Brea, USA). Glycated hemoglobin (HbAlc) was assessed by highperformance liquid chromatography (MQ-2000PT, Medconn, Shanghai, China). 


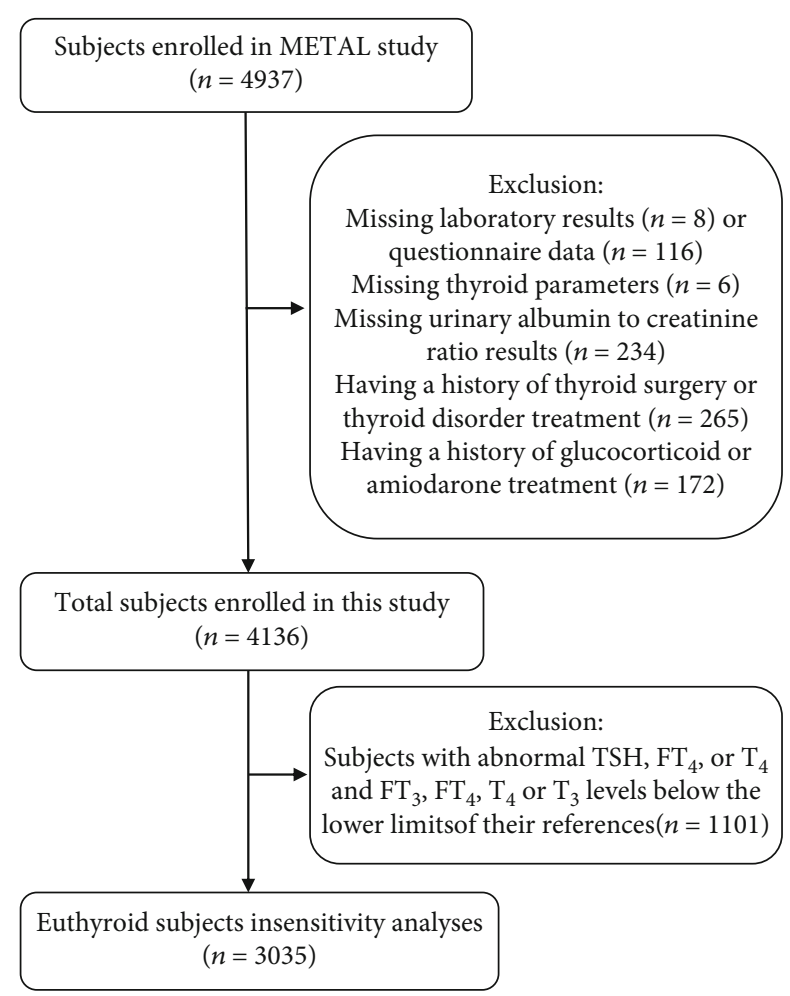

FIGURE 1: Flowchart of the inclusion and exclusion of participants.

2.3. Clinical Parameters. A questionnaire about sociodemographic characteristics, medical history, family history, and lifestyle factors was adopted during the interview. The same group of trained and experienced personnel in the SPECT-China study (Survey on Prevalence in East China for Metabolic Diseases and Risk Factors, ChiCTRECS-14005052, http://www.chictr.org.cn) [24, 25] conducted the interviews and clinical examinations, including measurements of weight, height, and blood pressure, according to a standard protocol. Body mass index (BMI) was calculated as weight in kilograms divided by height in meters squared. Current smoking was defined as having smoked at least 100 cigarettes in one's lifetime and currently smoking cigarettes [2]. Hypertension was assessed by systolic blood pressure $\geq 140 \mathrm{mmHg}$, diastolic blood pressure $\geq 90 \mathrm{mmHg}$, or self-reported previous diagnosis of hypertension by physicians or medication use for hypertension. Dyslipidemia was defined as TC $\geq 6.22 \mathrm{mmol} / \mathrm{L}, \mathrm{TG} \geq 2.26 \mathrm{mmol} / \mathrm{L}, \mathrm{LDL}-\mathrm{C} \geq$ $4.14 \mathrm{mmol} / \mathrm{L}, \mathrm{HDL}-\mathrm{C}<1.04 \mathrm{mmol} / \mathrm{L}$, self-reported previous diagnosis of hyperlipidemia by physicians, or medication use for dyslipidemia, according to the modified National Cholesterol Education Program-Adult Treatment Panel III.

2.4. Statistical Analysis. We performed survey analyses with IBM SPSS Statistics. All analyses were two-sided. A $P$ value $<0.05$ was taken to indicate a significant difference. Normally and nonnormally distributed continuous variables were expressed as mean $\pm \mathrm{SD}$ and median (interquartile range), respectively. Categorical variables are presented as percentages. Pearson's $\chi^{2}$ test was used for dichotomous variables. Associations among thyroid parameters and two kidney parameters (UACR and eGFR) were analyzed using linear regression models with each measure as the outcome. The regression models were adjusted for age, sex, $\mathrm{HbAlc}$, BMI, UA, the duration of diabetes, current smoking, dyslipidemia, hypertension, and the use of metformin. UACR and eGFR were $\ln$-transformed because of their skewed distribution. The results were expressed as $B$ values and 95\% confidence intervals (CIs). The relationship of thyroid hormones and parameters of thyroid homeostasis with reduced eGFR, higher UACR, and higher UACR and/or reduced eGFR (all categorical variables) were assessed by logistic regression. The regression models were adjusted for age, sex, HbAlc, BMI, UA, the duration of diabetes, current smoking, dyslipidemia, hypertension, and the use of metformin. The results were expressed as odds ratios (ORs) (95\% CIs). Area under the receiver operating characteristic curve (AUROC) analyses were used to compare the predictive powers of thyroid parameters for reduced eGFR, higher UACR, and higher UACR and/or reduced eGFR. Sensitivity analyses were performed in euthyroid participants with type 2 diabetes.

\section{Results}

3.1. Clinical Characteristics of the Participants. This study recruited 4136 participants with type 2 diabetes, with a mean age of 67.23 years old (SD 8.63, max 99, min 23); among them, 1983 were men. The clinical characteristics of all these participants are shown in Table 1 . The mean duration of diabetes of the participants was 9 years, and the mean level of HbAlc was $7.2 \%$. The prevalence of reduced eGFR, higher UACR, and higher UACR and/or reduced eGFR was 5.58\%, $26.0 \%$, and $28.1 \%$, respectively.

3.2. Prevalence of Kidney Disorders according to Thyroid Hormone Quartile Levels. Then, we analyzed the prevalence of reduced eGFR, higher UACR, and higher UACR and/or reduced eGFR according to the quartile levels of thyroid hormones (Figure 2). In all participants, we observed that the prevalence of reduced eGFR decreased with increasing levels of $\mathrm{FT}_{3}$ and $\mathrm{T}_{3}$ quartiles (both $P<0.001$ ). In terms of the prevalence of higher UACR and higher UACR and/or reduced eGFR, we found that they were negatively associated with quartiles of $\mathrm{FT}_{3}$ or $\mathrm{T}_{3}$ (both $P<0.001$ ) and were positively associated with quartiles of $\mathrm{FT}_{4}(P=0.029$ and 0.011, respectively). Although there was a significant difference between the prevalence of higher UACR and/or reduced eGFR and TSH levels, it was not linearly correlated but was instead a "U shape"; that is, the lowest and highest quartiles of TSH have a higher prevalence of higher UACR and/or reduced eGFR. We obtained similar results when we grouped the total participants according to TSH reference ranges $(<0.27 \mathrm{mIU} / \mathrm{L}, 0.27 \sim 4.20 \mathrm{mIU} / \mathrm{L}$, and $>4.2 \mathrm{mIU} / \mathrm{L})$. The prevalence of reduced eGFR was $6.7 \%, 5.0 \%$, and $8.0 \%(P=0.008)$; the prevalence of higher UACR was $53.3 \%, 25.0 \%$, and $31.0 \%(P<0.001)$; and the prevalence of higher UACR and/or reduced eGFR was $53.3 \%, 26.8 \%$, and $34.4 \%(P<0.001)$, respectively. 
TABLE 1: Clinical characteristics of all participants.

\begin{tabular}{|c|c|c|c|}
\hline & All & Men & Women \\
\hline$N$ & 4136 & 1983 & 2153 \\
\hline Age (yr) & $67.23 \pm 8.63$ & $67.50 \pm 8.66$ & $66.97 \pm 8.61$ \\
\hline Duration of diabetes ( $\mathrm{yr}$ ) & $9.00(12.00)$ & $10.00(12.00)$ & $8.00(12.00)$ \\
\hline Current smoking (\%) & 18.7 & 36.3 & 2.4 \\
\hline BMI $\left(\mathrm{kg} / \mathrm{m}^{2}\right)$ & $24.97 \pm 3.58$ & $24.99 \pm 3.29$ & $24.96 \pm 3.82$ \\
\hline HbAlc (\%) & $7.20(1.60)$ & $7.30(1.60)$ & $7.10(1.50)$ \\
\hline $\mathrm{UA}(\mu \mathrm{mol} / \mathrm{L})$ & $327.55 \pm 81.41$ & $343.79 \pm 82.08$ & $312.60 \pm 77.87$ \\
\hline Hypertension (\%) & 84.6 & 84.5 & 84.8 \\
\hline Dyslipidemia (\%) & 62.0 & 63.5 & 60.5 \\
\hline UACR (mg/g Cr) & $13.00(23.00)$ & $13.00(24.00)$ & $14.00(23.00)$ \\
\hline $\mathrm{eGFR}\left(\mathrm{ml} / \mathrm{min} / 1.73 \mathrm{~m}^{2}\right)$ & $95.63(18.59)$ & $94.56(18.64)$ & $96.66(18.02)$ \\
\hline Reduced eGFR (\%) & 5.58 & 6.0 & 5.1 \\
\hline Higher UACR (\%) & 26.0 & 25.9 & 26.2 \\
\hline Higher UACR and/or reduced eGFR (\%) & 28.1 & 27.8 & 28.4 \\
\hline \multicolumn{4}{|l|}{ Thyroid parameters } \\
\hline TSH (mIU/L) & $2.53(1.78)$ & $2.33(1.58)$ & $2.73(1.97)$ \\
\hline $\mathrm{FT}_{3}(\mathrm{pmol} / \mathrm{L})$ & $4.63 \pm 0.60$ & $4.78 \pm 0.60$ & $4.50 \pm 0.57$ \\
\hline $\mathrm{FT}_{4}(\mathrm{pmol} / \mathrm{L})$ & $16.76 \pm 2.29$ & $16.96 \pm 2.30$ & $16.58 \pm 2.26$ \\
\hline $\mathrm{T}_{3}(\mathrm{nmol} / \mathrm{L})$ & $1.65 \pm 0.29$ & $1.66 \pm 0.30$ & $1.64 \pm 0.28$ \\
\hline $\mathrm{T}_{4}(\mathrm{nmol} / \mathrm{L})$ & $101.05 \pm 17.84$ & $98.95 \pm 17.99$ & $102.99 \pm 17.49$ \\
\hline TPOAb positive (\%) & 7.7 & 5.2 & 10.0 \\
\hline TgAb positive (\%) & 6.2 & 3.3 & 9.0 \\
\hline \multicolumn{4}{|l|}{ Thyroid homeostasis parameters } \\
\hline SPINA-G ${ }_{D}(\mathrm{nmol} / \mathrm{s})$ & $15.33 \pm 3.12$ & $15.25 \pm 3.23$ & $15.41 \pm 3.02$ \\
\hline SPINA-G ${ }_{\mathrm{T}}(\mathrm{pmol} / \mathrm{s})$ & $2.31(1.05)$ & $2.38(1.05)$ & $2.25(1.06)$ \\
\hline TSHI & $3.17 \pm 0.58$ & $3.13 \pm 0.57$ & $3.22 \pm 0.59$ \\
\hline TTSI $(\mathrm{m} / \mathrm{L})$ & $192.32(132.76)$ & $179.96(122.53)$ & $207.08(143.21)$ \\
\hline
\end{tabular}

Normally and nonnormally distributed continuous variables were expressed as mean \pm SD and median (interquartile range), respectively. BMI: body mass index; HbAlc: glycated hemoglobin; UA: uric acid; UACR: urinary albumin to creatinine ratio; Cr: creatinine; eGFR: estimated glomerular filtration rate; TSH: thyroid-stimulating hormone; $\mathrm{FT}_{3}$ : free triiodothyronine; $\mathrm{FT}_{4}$ : free thyroxine; $\mathrm{T}_{4}$ : thyroxin; $\mathrm{T}_{3}$ : triiodothyronine; TPOAb: thyroid peroxidase antibody: TgAb: thyroglobulin antibody; SPINA- $\mathrm{G}_{\mathrm{D}}$ : sum activity of peripheral deiodinases; SPINA-G $\mathrm{G}_{\mathrm{T}}$ : thyroid's secretory capacity; TSHI: Jostel's TSH index; TTSI: thyrotroph thyroid hormone resistance index.

3.3. Association of Thyroid Parameters with UACR and eGFR. To more effectively reveal the relationship of thyroid parameters with two main important indicators (UACR and eGFR) of diabetic kidney status, linear regression analysis was used. Table 2 summarizes the results of the models. UACR and eGFR were both ln-transformed because of their skewed distribution. After adjusting for age, sex, the duration of diabetes, current smoking, HbAlc, UA, BMI, dyslipidemia, hypertension, and current use of metformin, UACR levels were negatively associated with the level of $\mathrm{FT}_{3}(B-0.171$; 95\% CI -0.242, -0.099; and $P<0.001)$ and $\mathrm{T}_{3}(B-0.269$; $95 \%$ CI $-0.408,-0.130$; and $P<0.001)$. In addition, in all participants, eGFR was positively associated with the level of $\mathrm{FT}_{3}$ (B 0.069; 95\% CI 0.0.057, 0.080; and $P<0.001)$ and $\mathrm{T}_{3}(B$ 0.096 ; $95 \%$ CI $0.074,0.118$; and $P<0.001)$ and was negatively associated with the level of TSH $(B-0.007 ; 95 \%$ CI -0.010 , -0.004; and $P<0.001), \mathrm{FT}_{4}(B-0.003 ; 95 \% \mathrm{CI}-0.006,0.000$; and $P=0.031)$, and $\mathrm{TgAb}$ positivity $(B-0.029 ; 95 \% \mathrm{CI}$
-0.056, -0.003; and $P=0.028$ ). Moreover, there was no other significant association of UACR and eGFR with thyroid parameters $(P>0.05)$.

3.4. Association of Thyroid Hormones and Antibodies with the Prevalence of Kidney Disorders. Given that thyroid status was associated with kidney disease risk factors (UACR and eGFR), we evaluated the adjusted odds ratios (ORs) for the prevalence of kidney disorder status (reduced eGFR, higher UACR, and higher UACR and/or reduced eGFR). Adjusted ORs were calculated after adjusting for age, sex, the duration of diabetes, current smoking, HbAlc, UA, BMI, dyslipidemia, hypertension, and current use of metformin using binary logistic regression models. As shown in Figure 3, higher TSH (OR 1.062; 95\% CI 1.004, 1.124; and $P=0.036$ ) and $\mathrm{FT}_{4}$ (OR 1.090; 95\% CI 1.020, 1.164; and $P=0.011$ ) and lower $\mathrm{FT}_{3}$ (OR 0.276; 95\% CI 0.200, 0.380; and $P<$ 0.001 ) and $\mathrm{T}_{3}$ (OR 0.119; 95\% CI 0.063, 0.226; and $P<$ 
Prevalence of reduced eGFR (total participants)

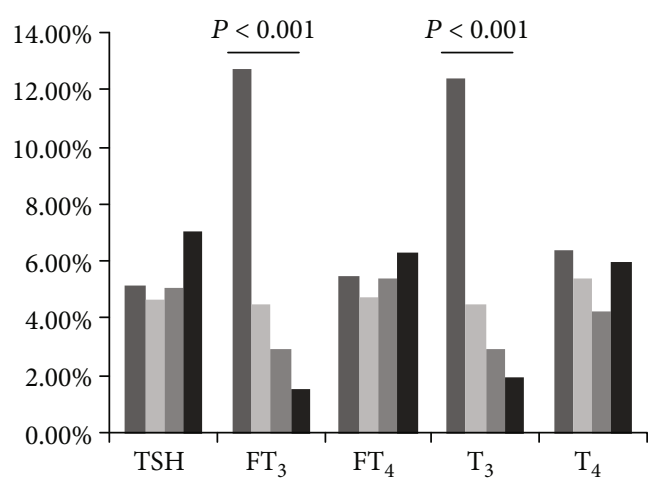

Prevalence of higher UACR (total participants)

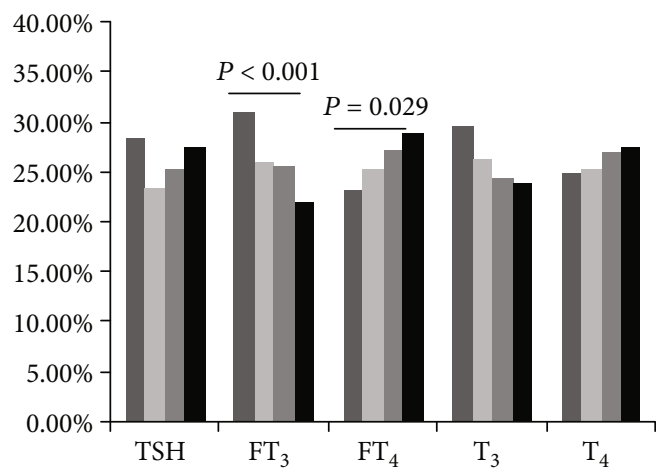

Prevalence of higher UACR and/orreduced eGFR (total participants)

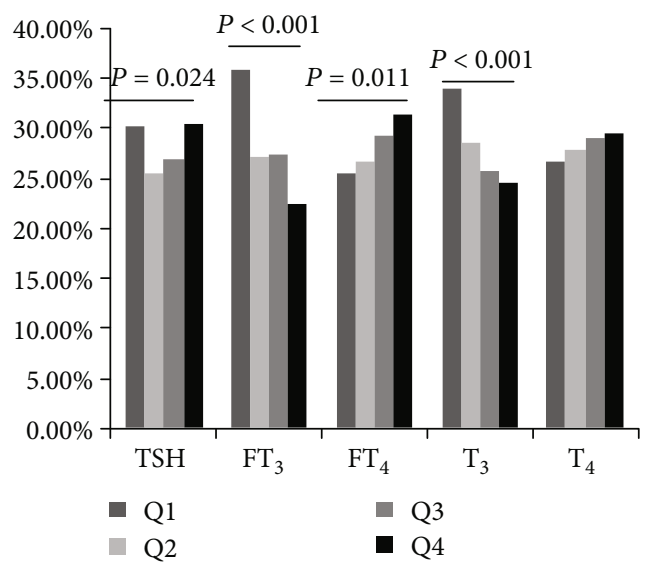

Prevalence of reduced eGFR (euthyroid participants)

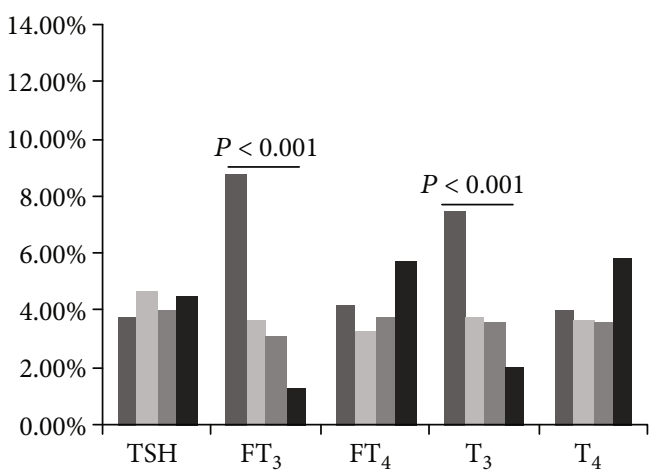

Prevalence of higher UACR (euthyroid participants)

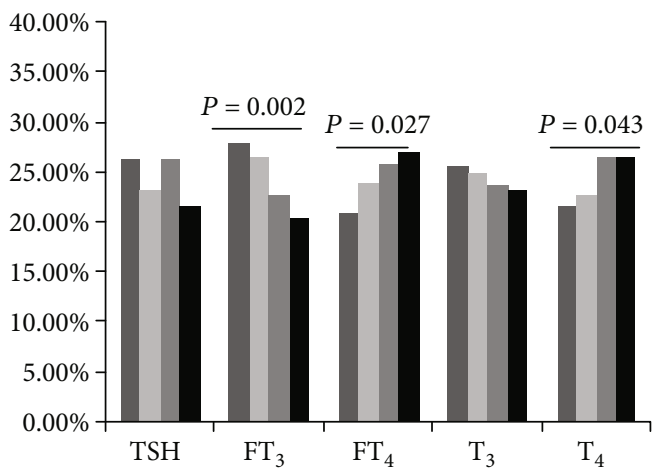

Prevalence of higher UACR and/orreduced eGFR (euthyroid participants)

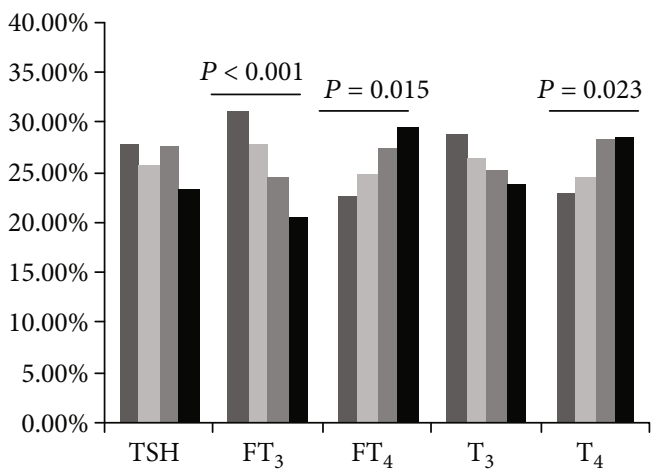

FIGURE 2: The prevalence of reduced eGFR, higher UACR, and higher UACR and/or reduced eGFR according to thyroid hormone quartile levels.

$0.001)$ were associated with higher prevalence of reduced eGFR. Higher $\mathrm{FT}_{4}$ (OR 1.038; 95\% CI 1.003, 1.075; and $P=$ 0.033 ) and lower $\mathrm{FT}_{3}$ (OR 0.796; 95\% CI 0.687, 0.922; and $P=0.002)$ and $\mathrm{T}_{3}$ (OR 0.682; 95\% CI 0.514, 0.906; and $P=$ $0.008)$ were associated with a higher prevalence of higher UACR. Similarly, higher $\mathrm{FT}_{4}$ (OR 1.050; 95\% CI 1.015, 1.086; and $P=0.005$ ) and lower $\mathrm{FT}_{3}$ (OR 0.739; 95\% CI $0.639,0.855$; and $P<0.001)$ and $\mathrm{T}_{3}$ (OR 0.593; 95\% CI $0.447,0.785$; and $P<0.001$ ) were associated with a higher prevalence of higher UACR and/or reduced eGFR. No thyroid antibodies were associated with kidney disorders.

We then compared the AUROC for reduced eGFR, higher UACR, and higher UACR and/or reduced eGFR between $\mathrm{TSH}, \mathrm{FT}_{3}, \mathrm{~T}_{3}, \mathrm{FT}_{4}$, and $\mathrm{T}_{4}$. We found that the AUROC for $\mathrm{FT}_{3}$ was 0.742 for reduced eGFR, 0.552 for higher UACR, and 0.571 for higher UACR and/or reduced eGFR, which was higher than other thyroid parameters (Table 3). Considering the efficiency of the AUROC 
TABLE 2: Association of thyroid parameters with UACR and eGFR by linear regression.

\begin{tabular}{|c|c|c|c|c|}
\hline & \multicolumn{2}{|c|}{$\ln U A C R$} & \multicolumn{2}{|c|}{ lneGFR } \\
\hline & $B(95 \% \mathrm{CI})$ & $P$ & $B(95 \% \mathrm{CI})$ & $P$ \\
\hline \multicolumn{5}{|l|}{ Total participants } \\
\hline TSH & $0.014(-0.004,0.033)$ & 0.136 & $-0.007(-0.010,-0.004)$ & $<0.001$ \\
\hline $\mathrm{FT}_{3}$ & $-0.171(-0.242,-0.099)$ & $<0.001$ & $0.069(0.057,0.080)$ & $<0.001$ \\
\hline $\mathrm{FT}_{4}$ & $0.008(-0.010,0.025)$ & 0.399 & $-0.003(-0.006,0.000)$ & 0.031 \\
\hline $\mathrm{T}_{3}$ & $-0.269(-0.408,-0.130)$ & $<0.001$ & $0.096(0.074,0.118)$ & $<0.001$ \\
\hline $\mathrm{T}_{4}$ & $0.000(-0.002,0.002)$ & 0.896 & $-0.000(-0.000,0.000)$ & 0.752 \\
\hline TPOAb positive & $0.092(-0.054,0.238)$ & 0.215 & $-0.023(-0.046,0.000)$ & 0.053 \\
\hline TgAb positive & $-0.004(-0.168,0.160)$ & 0.961 & $-0.029(-0.056,-0.003)$ & 0.028 \\
\hline \multicolumn{5}{|c|}{ Euthyroid participants } \\
\hline TSH & $-0.029(-0.080,0.021)$ & 0.258 & $-0.004(-0.011,0.003)$ & 0.304 \\
\hline $\mathrm{FT}_{3}$ & $-0.124(-0.214,-0.033)$ & 0.008 & $0.046(0.034,0.059)$ & $<0.001$ \\
\hline $\mathrm{FT}_{4}$ & $0.011(-0.012,0.033)$ & 0.354 & $-0.005(-0.008,-0.002)$ & 0.001 \\
\hline $\mathrm{T}_{3}$ & $-0.088(-0.269,0.092)$ & 0.337 & $0.045(0.020,0.070)$ & $<0.001$ \\
\hline $\mathrm{T}_{4}$ & $0.002(-0.001,0.004)$ & 0.264 & $-0.001(-0.001,0.000)$ & 0.002 \\
\hline TPOAb positive & $-0.005(-0.186,0.177)$ & 0.958 & $-0.004(-0.029,0.022)$ & 0.782 \\
\hline TgAb positive & $-0.024(-0.231,0.184)$ & 0.822 & $-0.031(-0.060,-0.002)$ & 0.033 \\
\hline
\end{tabular}

UACR and eGFR were ln-transformed for normal distribution before linear regression analysis. The regression models were adjusted for age, sex, HbAlc, BMI, urine acid, the duration of diabetes, current smoking, dyslipidemia, hypertension, and current use metformin.

( $>0.7$ ), we calculated only the cutoff values of $\mathrm{FT}_{3}$ and $\mathrm{T}_{3}$ for reduced eGFR; they were $4.38 \mathrm{pmol} / \mathrm{L}$ and $1.49 \mathrm{nmol} / \mathrm{L}$, respectively.

3.5. Association of Thyroid Homeostasis Parameters with Kidney Disorders. To reveal the relationship between thyroid homeostasis parameters and kidney disorders, we further evaluated the adjusted odds ratios (ORs) of SPINA-G ${ }_{D}$, SPINA$\mathrm{G}_{\mathrm{T}}$, TSHI, and TTSI for the prevalence of kidney disorders. Adjusted ORs were calculated. As shown in Table 4, lower SPINA- $G_{D}$ was significantly associated with these three kidney disorder statuses, and higher TSHI and TTSI were also significantly associated with reduced eGFR (all $P<0.05$ ).

3.6. Sensitivity Analyses. Further, we wanted to explore the relationship between thyroid parameters and reduced kidney function in participants with normal thyroid function and without euthyroid sick syndrome (ESS). Similar results were found for all participants.

Among these euthyroid participants, the prevalence of reduced eGFR, higher UACR, and higher UACR and/or reduced eGFR increased significantly along with decreasing levels of $\mathrm{FT}_{3}$ and/or $\mathrm{T}_{3}$. We also found that the prevalence of higher UACR and higher UACR and/or reduced eGFR increased with the levels of $\mathrm{FT}_{4}$ and $\mathrm{T}_{4}$ (Figure 2). UACR levels were negatively associated with the level of $\mathrm{FT}_{3}$, while eGFR was positively associated with the level of $\mathrm{FT}_{3}$ and $\mathrm{T}_{3}$ but was negatively associated with the level of $\mathrm{FT}_{4}, \mathrm{~T}_{4}$, and TgAb positivity (Table 2 ). In logistic analysis, we also found lower $\mathrm{FT}_{3}$ or $\mathrm{T}_{3}$ levels, but higher $\mathrm{FT}_{4}$ or $\mathrm{T}_{4}$ levels were associated with the increased risks of reduced eGFR and higher UACR and/or reduced eGFR. Higher UACR was only negatively associated with $\mathrm{FT}_{3}$ (Figure 3 ). The AUROC of $\mathrm{FT}_{3}$ was
0.698 for reduced eGFR, 0.548 for higher UACR, and 0.562 for higher UACR and/or reduced eGFR, which was higher than other thyroid parameters (Table 3 ). Regarding the association of thyroid homeostasis parameters with kidney disorders in euthyroid participants, it was found that only lower SPINA- $G_{D}$ was significantly associated with these three kidney disorder statuses (Table 4).

\section{Discussion}

Diabetic nephropathy, as one of the complications of diabetes, is related to the health and longevity of diabetic patients. The gold standard for the diagnosis of DKD is through kidney biopsy, which is difficult to achieve in a large population of samples. Several studies have suggested that morning UACR, as an important risk predictor for diabetic nephropathy and renal events that may provide prognostic information $[26,27]$, is an easy way to obtain the same information as a $24 \mathrm{~h}$ urine collection, which has been considered the gold standard $[26,28]$. Thus, in our study, we focused on two main indictors of CKD and DKD [29], reduced eGFR and higher UACR.

Metformin, as a first-line treatment for type 2 diabetes, was reported to have some effect on the hypothalamicpituitary-thyroid axis [30], and it was also associated with a decrease in the levels of TSH, possibly by enhancing the effects of thyroid hormones in the pituitary and activating adenosine monophosphate-activated protein kinase (AMPK) [31]. Thus, in our study, the current use of metformin was taken into account in all regression analyses.

In our study, among all participants, we found that the lowest and highest quartile levels of TSH had a higher prevalence of reduced eGFR, higher UACR, and higher UACR 


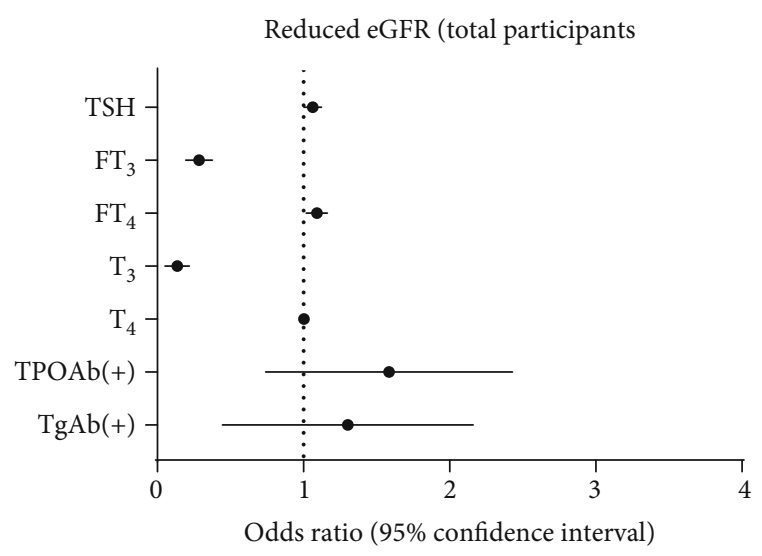

Higher UACR (total participants)

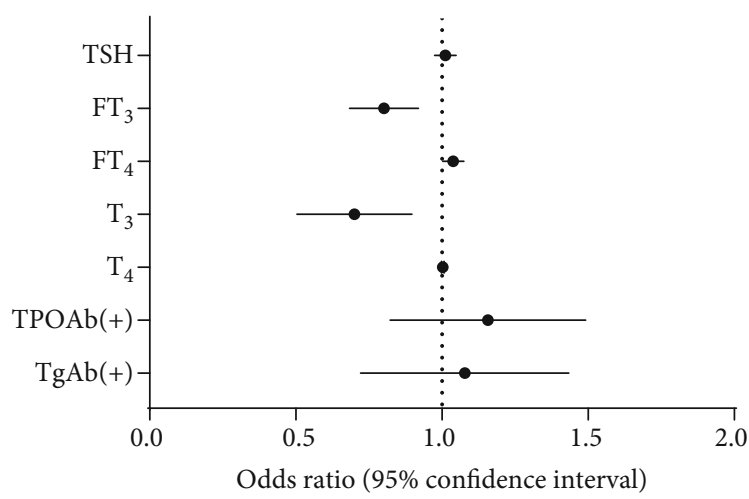

Higher UACR and/or reduced eGFR (total praticipants)

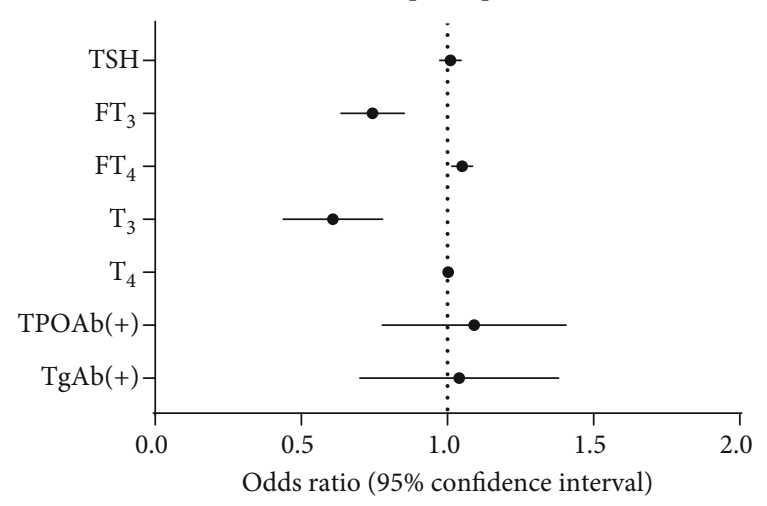

Reduced eGFR (euthyroid participants)

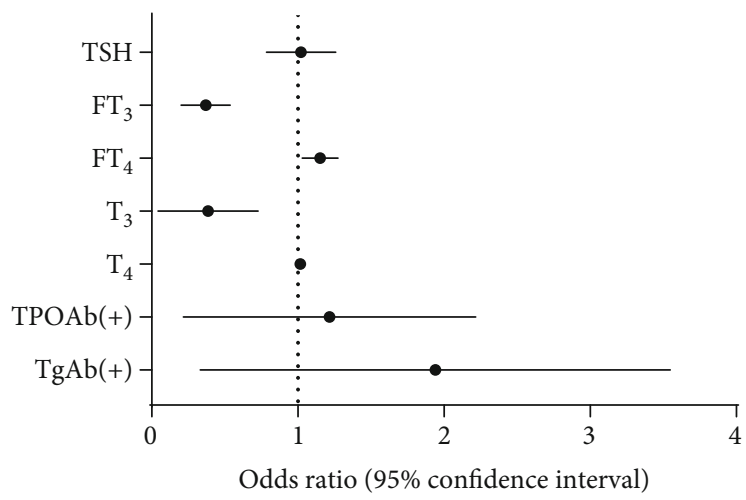

Higher UACR (euthyroid participants)

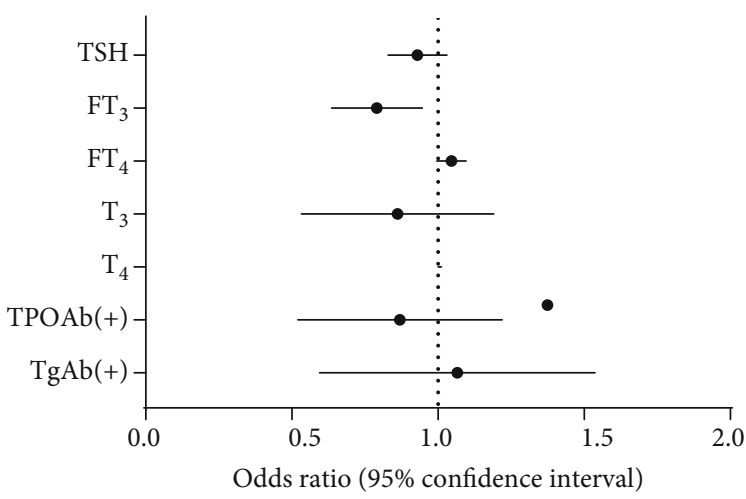

Higher UACR and/or reduced eGFR (euthyroid participants)

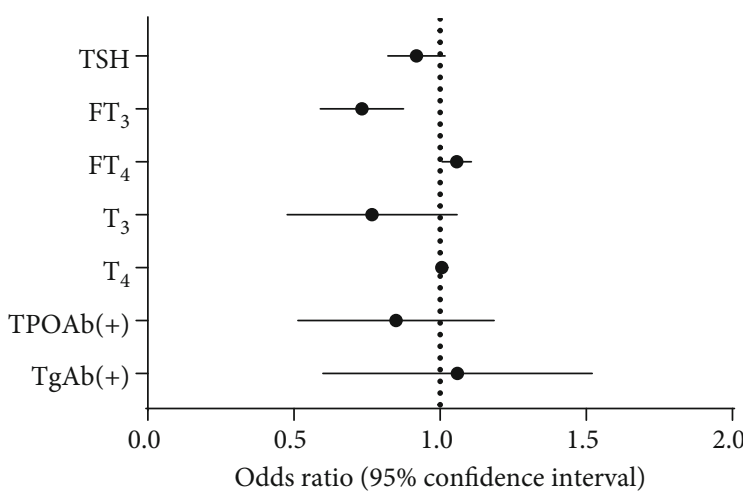

FIGURE 3: Associations of thyroid hormones and antibodies with reduced eGFR, higher UACR, and higher UACR and/or reduced eGFR. They were analyzed by using logistic regression. The regression models were adjusted for age, sex, HbA1c, BMI, UA, the duration of diabetes, current smoking, dyslipidemia, hypertension, and current use of metformin.

and/or reduced eGFR. Previous studies reported that both hypothyroidism and hyperthyroidism worsen kidney function directly by affecting renal blood flow, GFR, tubular function, electrolyte homeostasis, electrolyte pump functions, and kidney structure (e.g., decreased glomerular volume and area) and increasing peripheral vascular resistance [14-16], leading to higher serum CR concentrations and a lower eGFR. Several researchers also found an association of subclinical hypothyroidism with renal injury, such as macroalbuminuria in DM subjects [32-35]. Similarly, in our current study, the prevalence of these three kidney disorders was significantly different when all participants were grouped by the reference range of $\mathrm{TSH}$, the distribution of which looked like a "U" shape. Thus, we hypothesized that thyroid dysfunction, including overt and subclinical hypothyroidism or hyperthyroidism, was associated with kidney disorders in DM participants. On the other hand, it is worth pointing out that this association failed to be found when it was analyzed among participants with normal thyroid function in this study. Conflicting evidence has been 
TABLE 3: Comparison of AUROC between thyroid hormones for kidney disorders.

\begin{tabular}{|c|c|c|c|c|c|c|}
\hline & Reduced eGFR & $P$ & Higher UACR & $P$ & Higher UACR and/or reduced eGFR & $P$ \\
\hline \multicolumn{7}{|c|}{ Total participants } \\
\hline TSH & 0.549 & 0.014 & 0.502 & 0.881 & 0.506 & 0.517 \\
\hline $\mathrm{FT}_{3}$ & 0.742 & $<0.001$ & 0.552 & $<0.001$ & 0.571 & $<0.001$ \\
\hline $\mathrm{FT}_{4}$ & 0.521 & 0.276 & 0.528 & 0.007 & 0.531 & 0.002 \\
\hline $\mathrm{T}_{3}$ & 0.705 & $<0.001$ & 0.535 & 0.001 & 0.551 & $<0.001$ \\
\hline $\mathrm{T}_{4}$ & 0.486 & 0.479 & 0.515 & 0.130 & 0.515 & 0.123 \\
\hline \multicolumn{7}{|c|}{ Euthyroid participants } \\
\hline TSH & 0.518 & 0.490 & 0.486 & 0.241 & 0.486 & 0.242 \\
\hline $\mathrm{FT}_{3}$ & 0.698 & $<0.001$ & 0.548 & $<0.001$ & 0.562 & $<0.001$ \\
\hline $\mathrm{FT}_{4}$ & 0.544 & 0.093 & 0.529 & 0.018 & 0.533 & 0.006 \\
\hline $\mathrm{T}_{3}$ & 0.645 & $<0.001$ & 0.511 & 0.368 & 0.525 & 0.034 \\
\hline $\mathrm{T}_{4}$ & 0.542 & 0.104 & 0.535 & 0.004 & 0.536 & 0.002 \\
\hline
\end{tabular}

Data are presented as the area under the receiver operating characteristic curves (AUROC).

TABLE 4: Associations of thyroid homeostasis parameters with kidney disorders.

\begin{tabular}{|c|c|c|c|c|c|c|}
\hline & \multicolumn{2}{|c|}{ Reduced eGFR } & \multicolumn{2}{|c|}{ Higher UACR } & \multicolumn{2}{|c|}{ Higher UACR or reduced eGFR } \\
\hline & OR $(95 \% \mathrm{CI})$ & $P$ & OR (95\% CI) & $P$ & OR $(95 \% \mathrm{CI})$ & $P$ \\
\hline \multicolumn{7}{|c|}{ Total participants } \\
\hline SPINA- $G_{D}$ & $0.801(0.754,0.852)$ & $<0.001$ & $0.951(0.926,0.977)$ & $<0.001$ & $0.935(0.910,0.960)$ & $<0.001$ \\
\hline SPINA- $\mathrm{G}_{\mathrm{T}}$ & $0.993(0.937,1.052)$ & 0.814 & $1.013(0.994,1.032)$ & 0.187 & $1.012(0.994,1.030)$ & 0.197 \\
\hline TSHI & $1.349(1.021,1.783)$ & 0.035 & $1.027(0.896,1.178)$ & 0.703 & $1.050(0.917,1.202)$ & 0.479 \\
\hline TTSI & $1.001(1.000,1.002)$ & 0.018 & $1.000(1.000,1.001)$ & 0.294 & $1.000(1.000,1.001)$ & 0.276 \\
\hline \multicolumn{7}{|c|}{ Euthyroid participants } \\
\hline SPINA-G $\mathrm{D}_{\mathrm{D}}$ & $0.831(0.755,0.913)$ & $<0.001$ & $0.961(0.926,0.996)$ & 0.031 & $0.947(0.914,0.982)$ & 0.003 \\
\hline SPINA-G $\mathrm{G}_{\mathrm{T}}$ & $1.054(0.866,1.283)$ & 0.601 & $1.064(0.973,1.162)$ & 0.173 & $1.065(0.976,1.162)$ & 0.157 \\
\hline TSHI & $1.449(0.934,2.248)$ & 0.098 & $1.000(0.821,1.218)$ & 0.999 & $1.017(0.837,1.235)$ & 0.866 \\
\hline TTSI & $1.000(1.000,1.000)$ & 0.437 & $1.000(1.000,1.000)$ & 0.538 & $1.000(1.000,1.000)$ & 0.501 \\
\hline
\end{tabular}

They were analyzed by using logistic regression. The regression models were adjusted for age, sex, HbAlc, BMI, UA, the duration of diabetes, current smoking, dyslipidemia, hypertension, and current use of metformin.

reported about the association of TSH and kidney status in euthyroid DM subjects $[14,36,37]$. The HUNT study reported that $\mathrm{TSH}$ within the reference range was negatively associated with eGFR [14] in type 2 diabetes, while others $[7,38]$ reached a different conclusion.

In terms of other thyroid hormones, we observed increased prevalence of reduced eGFR, higher UACR, and higher UACR and/or reduced eGFR along with decreased levels of $\mathrm{FT}_{3}$ and $\mathrm{T}_{3}$. After full adjustment, $\mathrm{FT}_{3}$ and $\mathrm{T}_{3}$ levels were significantly negatively associated with UACR and were positively associated with eGFR. In logistic analysis, lower $\mathrm{FT}_{3}$ and $\mathrm{T}_{3}$ were also found to be associated with increased risks of reduced eGFR, higher UACR, and higher UACR and/or reduced eGFR. Furthermore, though we found that the AUROC of $\mathrm{FT}_{3}$ for these three patterns of kidney disorders was much higher than that for any other thyroid hormones, only the AUROC of $\mathrm{FT}_{3}$ and $\mathrm{T}_{3}$ for reduced eGFR in all participants was larger than 0.7 , which may give some significant values. The cutoff values of lower $\mathrm{FT}_{3}$ and $\mathrm{T}_{3}$ for reduced eGFR were $4.38 \mathrm{pmol} / \mathrm{L}$ and $1.49 \mathrm{nmol} / \mathrm{L}$, respectively. These results were in agreement with some other cohort and cross-sectional studies that suggested that lower
$\mathrm{T}_{3}$ has been consistently found to be the most common disturbance in patients with kidney disorders and that it was an independent predictor of survival in various illness states $[12,36,39-42]$. However, only a few studies have focused on the relationship between normal thyroid hormone levels and renal events in DM participants. In our present study, these results were strongly consistent even though $\mathrm{FT}_{3}$ and $\mathrm{T}_{3}$ levels were in the normal reference range. In addition, $\mathrm{FT}_{3}$ but not $\mathrm{FT}_{4}$ was reduced in kidney disorders, suggesting that low $\mathrm{FT}_{3}$ is unlikely to be an in vitro artifact because substances inhibiting binding are expected to affect $\mathrm{FT}_{4}$ to a greater extent than $\mathrm{FT}_{3}$. Thus, reduced $\mathrm{FT}_{3}$ seems to reflect a true selective $T_{3}$ deficiency due to the inhibition of $5^{\prime}$ -deiodinase, which is a catalyzing enzyme for the production of $\mathrm{T}_{3}$ from circulating $\mathrm{T}_{4}[39,43]$. Higher $\mathrm{FT}_{4}$ and lower $\mathrm{T}_{3}$ concentrations were reported to be strongly and independently associated with renal events [44]. In this study, we obtained the same results.

It has been suggested that autoimmune thyroid disease may lead to the deposition of immunocomplexes in the renal glomeruli and may be responsible for endothelial dysfunction and subsequent microalbuminuria [45]. In the present 
study, both among all participants and among euthyroid participants, $\operatorname{TgAb}$ positivity was significantly associated with reduced eGFR, which suggested that thyroid autoimmunity may be involved in renal injury. However, there are different points of view. Suher et al. reported that the association with eGFR was roughly similar for hypothyroidism with and without TPOAb positivity, which suggested that hypothyroidismassociated kidney dysfunction seems to be more related to the decline in thyroid hormone levels than to thyroid autoimmunity [46].

Finally, we explored the relationship of thyroid homeostasis parameters, including two structural parameters of thyroid homeostasis (SPINA- $\mathrm{G}_{\mathrm{D}}$ and SPINA- $\mathrm{G}_{\mathrm{T}}$ ) and two pituitary thyrotropic function indices (TSHI and TTSI), with kidney status. By extending the classic concept of separate measurements of thyroid hormone parameters, these markers add new qualitative and quantitative dimensions to the evaluation of thyroid homeostasis, offer a more integrated and systemic view, and deliver important insights into the physiology of pituitary-thyroid feedback control [20]. Though it was reported that SPINA- $\mathrm{G}_{\mathrm{T}}$ was correlated with creatinine clearance $[20,21]$, we found that lower SPINA- $G_{D}$ was significantly associated with three kidney disorder statuses, and higher TSHI and TTSI were significantly associated with reduced eGFR. Therefore, it was suggested that kidney disorders may be related to the maximum stimulated activity of step-up deiodination and pituitary thyrotropic function.

Though our study had some strengths, including novelty, the analysis of community dwelling participants with a relatively large sample size, and strong quality control, there were also some limitations. First, because of the cross-sectional study, we can evaluate association but not causality. We conducted a Mendelian randomization (MR) analysis to examine the causality of the association between TSH and eGFR in 10603 participants recruited from the Survey on Prevalence in East China for Metabolic Diseases and Risk Factors (SPECT-China, ChiCTR-ECS-14005052, http://www.chictr .org.cn) $[25,47]$. By a genetic approach that limits residual confounding and reverse causation in observational conventional epidemiological studies, TSH and eGFR were not causally associated, which suggested that genetically elevated TSH concentrations may not affect renal function [48]. The mechanism linking kidney function and thyroid homeostasis remains unclear. Thyroid dysfunction may trigger kidney failure, and renal failure might trigger an allostatic response of thyroid function as well, i.e., euthyroid sick syndrome [49] or thyroid allostasis in critical illness, tumors, uremia, and starvation (TACITUS) [50], albeit in slight form. This is known from previous studies and is plausible because the kidney expresses deiodinases that catalyze the conversion of $\mathrm{T}_{4}$ to $\mathrm{T}_{3}$. Further prospective and longitudinal studies are needed. Second, UACR and thyroid function were estimated only once, which could result in misleading classifications. Third, the levels of $\mathrm{rT}_{3}$ were not measured in this study.

\section{Conclusion}

Among type 2 diabetes patients, elevated TSH and $\mathrm{FT}_{4}$ (or $\mathrm{T}_{4}$ ), lower $\mathrm{FT}_{3}\left(\right.$ or $\mathrm{T}_{3}$ ), and $\mathrm{TgAb}$ positivity were associated with higher UACR and lower eGFR levels. Elevated TSH and $\mathrm{FT}_{4}\left(\right.$ or $\left.\mathrm{T}_{4}\right)$, lower $\mathrm{FT}_{3}\left(\right.$ or $\mathrm{T}_{3}$ ) levels, and thyroid homeostasis parameters (SPINA- $\mathrm{G}_{\mathrm{D}}$, TSHI, and TTSI) were associated with the prevalence of kidney disorders. The lower $\mathrm{FT}_{3}$, even within a normal reference range, was the factor most related to reduced eGFR compared with all other thyroid hormones. Multidisciplinary collaborative care, including nephrologists, endocrinologists, or internal medicine specialists, is needed and appears to positively impact the clinical, humanistic, and economic outcomes of patients with diabetes [51, 52].

\section{Abbreviations}

CKD: $\quad$ Chronic kidney disease

DKD: Diabetic kidney disease

TH: $\quad$ Thyroid hormone

UACR: Urinary albumin to creatinine ratio

CR: Creatinine

eGFR: $\quad$ Estimated glomerular filtration rate

TSH: Thyroid-stimulating hormone

$\mathrm{FT}_{3}$ : $\quad$ Free triiodothyronine

$\mathrm{FT}_{4}$ : $\quad$ Free thyroxine

$\mathrm{T}_{4}$ : $\quad$ Thyroxin

$\mathrm{T}_{3}$ : $\quad$ Triiodothyronine

TPOAb: Thyroid peroxidase antibody

TgAb: Thyroglobulin antibody

BMI: $\quad$ Body mass index

HbA1c: Glycated hemoglobin

CAP: $\quad$ College of American Pathologists

CKD-EPI: Chronic Kidney Disease Epidemiology Collaboration

TG: $\quad$ Triglycerides

TC: $\quad$ Total cholesterol

HDL-C: High-density lipoprotein

LDL-C: Low-density lipoprotein

UA: $\quad$ Uric acid

AUROC: Area under the receiver operating characteristic curve

ORs: Odds ratios

SPINA-G $\mathrm{D}_{\mathrm{D}}$ : Sum activity of peripheral deiodinases

SPINA-G $\mathrm{G}_{\mathrm{T}}$ : Thyroid's secretory capacity

TSHI: Jostel's TSH index

TTSI: Thyrotroph thyroid hormone resistance index.

\section{Data Availability}

The data used to support the findings of this study are included within the article.

\section{Disclosure}

The funders played no role in the design or conduct of the study, collection, management, analysis, or interpretation of data or in the preparation, review, or approval of the article. 


\section{Conflicts of Interest}

The authors declare that they have no competing interests.

\section{Authors' Contributions}

Yingli Lu designed, performed, and supervised this investigation and had full access to all of the data and took responsibility for the integrity of the data and the accuracy of the data analysis. Yi Chen and Wen Zhang contributed equally to this work. They performed this investigation; analyzed the data; and contributed to the discussion, interpretation of the data, and the manuscript writing. Ningjian Wang, Yuying Wang, Chiyu Wang, and Heng Wan provided technical or material support and contributed to the discussion. All authors read and approved the final manuscript.

\section{Acknowledgments}

This study was supported by the National Natural Science Foundation of China (81800694, 91857117), Science and Technology Commission of Shanghai Municipality (19140902400, 18410722300), Major Science and Technology Innovation Program of Shanghai Municipal Education Commission (2019-01-07-00-01-E00059), Commission of Health and Family Planning of Pudong District (PWZxq2017-17), Municipal Human Resources Development Program for Outstanding Young Talents in Medical and Health Sciences in Shanghai (2017YQ053), and Shanghai Jiao Tong University School of Medicine (19XJ11007).

\section{References}

[1] N. H. Cho, J. E. Shaw, S. Karuranga et al., "IDF diabetes atlas: global estimates of diabetes prevalence for 2017 and projections for 2045," Diabetes Research and Clinical Practice, vol. 138, pp. 271-281, 2018.

[2] Y. Xu, L. Wang, J. He et al., "Prevalence and control of diabetes in Chinese adults," Journal of the American Medical Association, vol. 310, no. 9, pp. 948-959, 2013.

[3] P. Stenvinkel, "Chronic kidney disease: a public health priority and harbinger of premature cardiovascular disease," Journal of Internal Medicine, vol. 268, no. 5, pp. 456-467, 2010.

[4] V. Jha, G. Garcia-Garcia, K. Iseki et al., "Chronic kidney disease: global dimension and perspectives," The Lancet, vol. 382, no. 9888, pp. 260-272, 2013.

[5] J. Coresh, E. Selvin, L. A. Stevens et al., "Prevalence of chronic kidney disease in the United States," Journal of the American Medical Association, vol. 298, no. 17, pp. 2038-2047, 2007.

[6] S. I. Hallan, K. Matsushita, Y. Sang et al., "Age and association of kidney measures with mortality and end-stage renal disease," Journal of the American Medical Association, vol. 308, no. 22, pp. 2349-2360, 2012.

[7] J. Zou, F. Tian, Y. Zhang et al., "Association between thyroid hormone levels and diabetic kidney disease in euthyroid patients with type 2 diabetes," Scientific Reports, vol. 8, no. 1, article 4728, 2018.

[8] American Diabetes Association, "Nephropathy in diabetes," Diabetes Care, vol. 27, Supplement 1, pp. S79-S83, 2004.

[9] A. Levin and P. E. Stevens, "Summary of KDIGO 2012 CKD guideline: behind the scenes, need for guidance, and a frame- work for moving forward," Kidney International, vol. 85, no. 1, pp. 49-61, 2014.

[10] J. Gattineni, D. Sas, A. Dagan, V. Dwarakanath, and M. Baum, "Effect of thyroid hormone on the postnatal renal expression of NHE8," American Journal of Physiology. Renal Physiology, vol. 294, no. 1, pp. F198-F204, 2008.

[11] C. L. Meuwese and J. J. Carrero, "Chronic kidney disease and hypothalamic-pituitary axis dysfunction: the chicken or the egg?," Archives of Medical Research, vol. 44, no. 8, pp. 591600, 2013.

[12] P. Iglesias and J. J. Diez, "Thyroid dysfunction and kidney disease," European Journal of Endocrinology, vol. 160, no. 4, pp. 503-515, 2009.

[13] M. Mohamedali, S. Reddy Maddika, A. Vyas, V. Iyer, and P. Cheriyath, "Thyroid disorders and chronic kidney disease," International Journal of Nephrology, vol. 2014, Article ID 520281, 6 pages, 2014.

[14] B. O. Asvold, T. Bjoro, and L. J. Vatten, "Association of thyroid function with estimated glomerular filtration rate in a population-based study: the HUNT study," European Journal of Endocrinology, vol. 164, no. 1, pp. 101-105, 2011.

[15] L. H. Mariani and J. S. Berns, "The renal manifestations of thyroid disease," Journal of the American Society of Nephrology, vol. 23, no. 1, pp. 22-26, 2012.

[16] J. G. den Hollander, R. W. Wulkan, M. J. Mantel, and A. Berghout, "Correlation between severity of thyroid dysfunction and renal function," Clinical Endocrinology, vol. 62, no. 4, pp. 423-427, 2005.

[17] C. Wang, W. Zhang, Y. Wang et al., "Novel associations between sex hormones and diabetic vascular complications in men and postmenopausal women: a cross-sectional study," Cardiovascular Diabetology, vol. 18, no. 1, p. 97, 2019.

[18] N. Wang, Y. Wang, W. Zhang et al., "C-peptide is associated with NAFLD inflammatory and fibrotic progression in type 2 diabetes," Diabetes/Metabolism Research and Reviews, vol. 36, no. 2, article e3210, 2019.

[19] D. Rosolowska-Huszcz, L. Kozlowska, and A. Rydzewski, "Influence of low protein diet on nonthyroidal illness syndrome in chronic renal failure," Endocrine, vol. 27, no. 3, pp. 283-288, 2005.

[20] J. W. Dietrich, G. Landgrafe-Mende, E. Wiora et al., "Calculated parameters of thyroid homeostasis: emerging tools for differential diagnosis and clinical research," Frontiers in Endocrinology, vol. 7, p. 57, 2016.

[21] A. Jostel, W. D. Ryder, and S. M. Shalet, "The use of thyroid function tests in the diagnosis of hypopituitarism: definition and evaluation of the TSH index," Clinical Endocrinology, vol. 71, no. 4, pp. 529-534, 2009.

[22] L. A. Stevens, M. A. Claybon, C. H. Schmid et al., "Evaluation of the Chronic Kidney Disease Epidemiology Collaboration equation for estimating the glomerular filtration rate in multiple ethnicities," Kidney International, vol. 79, no. 5, pp. 555562, 2011.

[23] American Diabetes Association, "10. microvascular complications and foot care: standards of medical care in diabetes-2018," Diabetes Care, vol. 41, Supplement 1, pp. S105-S118, 2018.

[24] N. Wang, Y. Chen, Z. Ning et al., "Exposure to famine in early life and nonalcoholic fatty liver disease in adulthood," The Journal of Clinical Endocrinology \& Metabolism, vol. 101, no. 5, pp. 2218-2225, 2016. 
[25] Y. Chen, Y. Chen, F. Xia et al., "A higher ratio of estradiol to testosterone is associated with autoimmune thyroid disease in males," Thyroid, vol. 27, no. 7, pp. 960-966, 2017.

[26] P. Rossing, "Assessment of kidney function: UACR-two risk markers or a $24 \mathrm{~h}$ collection in a minute?," Nature Reviews Nephrology, vol. 8, no. 6, pp. 320-321, 2012.

[27] K. Rossing, H. Mischak, M. Dakna et al., "Urinary proteomics in diabetes and CKD," Journal of the American Society of Nephrology, vol. 19, no. 7, pp. 1283-1290, 2008.

[28] H. J. Lambers Heerspink, R. T. Gansevoort, B. M. Brenner et al., "Comparison of different measures of urinary protein excretion for prediction of renal events," Journal of the American Society of Nephrology, vol. 21, no. 8, pp. 1355-1360, 2010.

[29] L. Lobato and A. Rocha, "Transthyretin amyloidosis and the kidney," Clinical Journal of the American Society of Nephrology, vol. 7, no. 8, pp. 1337-1346, 2012.

[30] R. Krysiak, W. Szkrobka, and B. Okopien, "Effect of metformin on hypothalamic-pituitary-thyroid axis activity in elderly antipsychotic-treated women with type 2 diabetes and subclinical hypothyroidism: a preliminary study," Journal of Clinical Pharmacology, vol. 58, no. 5, pp. 586-592, 2018.

[31] X. Meng, S. Xu, G. Chen, M. Derwahl, and C. Liu, "Metformin and thyroid disease," The Journal of Endocrinology, vol. 233, no. 1, pp. R43-R51, 2017.

[32] S. Furukawa, S. Yamamoto, Y. Todo et al., "Association between subclinical hypothyroidism and diabetic nephropathy in patients with type 2 diabetes mellitus," Endocrine Journal, vol. 61, no. 10, pp. 1011-1018, 2014.

[33] L. Zhang, G. Yang, Z. Su, and J. Yang, "Correlation between subclinical hypothyroidism and renal function in patients with diabetes mellitus," Nephrology, vol. 22, no. 10, pp. 790-795, 2017.

[34] N. Mansournia, S. Riyahi, S. Tofangchiha et al., "Subclinical hypothyroidism and diabetic nephropathy in Iranian patients with type 2 diabetes," Journal of Endocrinological Investigation, vol. 40, no. 3, pp. 289-295, 2017.

[35] J. B. Zhou, H. B. Li, X. R. Zhu, H. L. Song, Y. Y. Zhao, and J. K. Yang, "Subclinical hypothyroidism and the risk of chronic kidney disease in T2D subjects: a case-control and dose-response analysis," Medicine, vol. 96, article e6519, 2017.

[36] Y. Zhang, Y. Chang, S. Ryu et al., "Thyroid hormone levels and incident chronic kidney disease in euthyroid individuals: the Kangbuk Samsung Health Study," International Journal of Epidemiology, vol. 43, no. 5, pp. 1624-1632, 2014.

[37] C. L. Meuwese, J. Gussekloo, A. J. de Craen, F. W. Dekker, and W. P. den Elzen, "Thyroid status and renal function in older persons in the general population," The Journal of Clinical Endocrinology and Metabolism, vol. 99, no. 8, pp. 2689-2696, 2014.

[38] J. Wu, X. Li, Y. Tao, Y. Wang, and Y. Peng, "Free triiodothyronine levels are associated with diabetic nephropathy in euthyroid patients with type 2 diabetes," International Journal of Endocrinology, vol. 2015, Article ID 204893, 7 pages, 2015.

[39] C. Zoccali, F. Mallamaci, G. Tripepi, S. Cutrupi, and P. Pizzini, "Low triiodothyronine and survival in end-stage renal disease," Kidney International, vol. 70, no. 3, pp. 523528, 2006.

[40] G. Iervasi, A. Pingitore, P. Landi et al., "Low-T3 syndrome: a strong prognostic predictor of death in patients with heart disease," Circulation, vol. 107, no. 5, pp. 708-713, 2003.
[41] J. C. Lo, G. M. Chertow, A. S. Go, and C. Y. Hsu, "Increased prevalence of subclinical and clinical hypothyroidism in persons with chronic kidney disease," Kidney International, vol. 67, no. 3, pp. 1047-1052, 2005.

[42] S. H. Song, I. S. Kwak, D. W. Lee, Y. H. Kang, E. Y. Seong, and J. S. Park, "The prevalence of low triiodothyronine according to the stage of chronic kidney disease in subjects with a normal thyroid-stimulating hormone," Nephrology, Dialysis, Transplantation, vol. 24, no. 5, pp. 1534-1538, 2009.

[43] K. D. Burman and L. Wartofsky, "Thyroid function in the intensive care unit setting," Critical Care Clinics, vol. 17, no. 1, pp. 43-57, 2001.

[44] U. T. Schultheiss, N. Daya, M. E. Grams et al., “Thyroid function, reduced kidney function and incident chronic kidney disease in a community-based population: the Atherosclerosis Risk in Communities study," Nephrology, Dialysis, Transplantation, vol. 32, pp. 1874-1881, 2017.

[45] G. D. Xiang, Y. S. He, L. S. Zhao, J. Hou, L. Yue, and H. J. Xiang, "Impairment of endothelium-dependent arterial dilation in Hashimoto's thyroiditis patients with euthyroidism," Clinical Endocrinology, vol. 64, no. 6, pp. 698-702, 2006.

[46] M. Suher, E. Koc, N. Ata, and C. Ensari, "Relation of thyroid disfunction, thyroid autoantibodies, and renal function," Renal Failure, vol. 27, no. 6, pp. 739-742, 2005.

[47] N. Wang, K. Zhang, B. Han et al., "Follicle stimulating hormone, its novel association with sex hormone binding globulin in men and postmenopausal women," Endocrine, vol. 56, no. 3, pp. 649-657, 2017.

[48] C. Chen, F. Xia, Y. Chen et al., "Association between thyroidstimulating hormone and renal function: a Mendelian randomization study," Kidney \& Blood Pressure Research, vol. 43, no. 4, pp. 1121-1130, 2018.

[49] I. J. Chopra, "Clinical review 86: euthyroid sick syndrome: is it a misnomer?," The Journal of Clinical Endocrinology and Metabolism, vol. 82, no. 2, pp. 329-334, 1997.

[50] J. W. Dietrich, G. Landgrafe, and E. H. Fotiadou, “A Higher Ratio of Estradiol to Testosterone Is Associated with Autoimmune Thyroid Disease in Males," Journal of Thyroid Research, vol. 27, no. 7, pp. 960-966, 2012.

[51] R. Minutolo, F. C. Sasso, P. Chiodini et al., "Management of cardiovascular risk factors in advanced type 2 diabetic nephropathy: a comparative analysis in nephrology, diabetology and primary care settings," Journal of Hypertension, vol. 24, no. 8, pp. 1655-1661, 2006.

[52] M. Y. L. Siaw and J. Y. Lee, "Multidisciplinary collaborative care in the management of patients with uncontrolled diabetes: a systematic review and meta-analysis," International Journal of Clinical Practice, vol. 73, no. 2, article e13288, 2019. 